\title{
Biomechanical Model Construction from Different Modalities: Application to Cardiac Images
}

\author{
M. Sermesant*, C. Forest, X. Pennec, H. Delingette, and N. Ayache \\ Epidaure Research Project, INRIA Sophia Antipolis \\ 2004 route des Lucioles, 06902 Sophia Antipolis, France \\ ${ }^{\star}$ Corresponding author: Maxime.Sermesant@inria.fr
}

\begin{abstract}
This article describes a process to include in a volumetric model various anatomical and mechanical information provided by different sources. Three stages are described, namely a mesh construction, the non-rigid deformation of the tetrahedral mesh into various volumetric images, and the rasterization procedure allowing the transfer of properties from a voxel grid to a tetrahedral mesh. The method is experimented on various imaging modalities, demonstrating its feasibility. By using a biomechanical model, we include physically-based a priori knowledge which should allow to better recover the cardiac motion from images.
\end{abstract}

\section{Introduction}

In a previous publication [19], we presented an active electro-mechanical model of the heart triggered by the electrocardiogram. The future objective of this model is the automatic segmentation of temporal sequences of cardiac images and the automatic extraction of functional parameters useful for the diagnosis and for patient's follow up [1]. This work was initially motivated by the seminal work of McCulloch et al. on the construction of electro-mechanical models of the heart [1] and the use of myocardium mechanical properties in a segmentation process by Papademetris and Duncan [15].

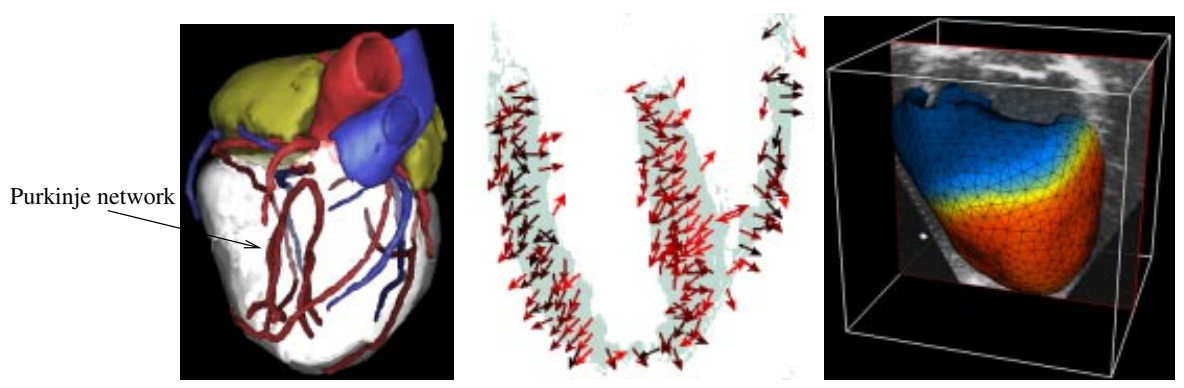

Fig. 1. Left: isosurfaces extracted from the segmented heart of the Visible Human Project. Middle: diffusion tensor image to define fiber directions. Right: the electromechanical model of the heart in a 3D ultrasound image.

An intermediate stage to reach this objective is the ability to include in the model various anatomical and mechanical information provided by different 
sources. An example is the introduction of the precise location of the Purkinje network, which provides important information on the sources of the electrical impulse in the myocardium. Another example is the knowledge of the fiber directions, which create dramatic mechanical and electrical local anisotropy. A last example is the knowledge of interactions between the organ and nearby structures, which can be modeled by fixed points at specific locations for instance.

Once the model is equipped with the required attributes, it is then possible to use a subset (or all) of these properties to guide its deformation in the images of a studied patient. This last stage is different but connected to other approaches, which also propose to use biomechanically controled meshes to compute deformations $6[9 \mid 2[5]$.

The process of building a biomechanical heart model can be decomposed into three independent tasks. First, a geometric mesh consisting of a set of tetrahedra is created from a volumetric image or from any other dataset. Second, this mesh must be registered to a given image modality (such as Diffusion Tensor Imaging, MR Imaging or histology reconstruction). Finally, the third stage consists in retrieving the information of interest (fiber directions, anatomical regions,...) from the volumetric image to the volumetric mesh. Since, we are using different image modalities, several registration and information retrieval tasks may be performed. The next three sections detail each of these three stages.

\section{Volumetric Mesh Creation}

We created a volumetric mesh of the heart that includes both right and left ventricles. Indeed, many image modalities have a field of view large enough to include both ventricles. Furthermore, the right ventricle motion provides also information that are relevant clinically. We have chosen to use a geometric representation based on tetrahedra rather than hexahedra (as proposed in 11]) in order to better capture the important geometric details of the RV and LV during the image segmentation stage. Moreover, tetrahedral meshes allow to perform local mesh refinement in a straightforward manner, whereas one must use more sophisticated hierarchical adaptive refinement of basis functions for achieving the same results on hexahedral meshes. However, it is widely accepted that hexahedral finite elements are better suited than tetrahedral elements for the deformation computation of incompressible materials.

When creating a tetrahedral mesh, one must take into account two parameters. First the mesh size should be small enough in order to keep the computation time compatible with user interaction. Second, the shape quality of tetrahedra must be high enough in order to produce accurate results. Our approach has been to create a triangular mesh of the ventricles as the result of a several low-level tasks: image segmentation, morphological operations, image smoothing, connected component extraction, isosurfacing and mesh decimation. The quality of triangular elements and their sampling (increased at parts of high curvature) was controlled visually. The tetrahedral mesh was generated with this triangular shell using the commercial software GHs3D 1 developed at INRIA. The resulting mesh has 2000 vertices and 10000 tetrahedra.

\footnotetext{
${ }^{1}$ http://www-rocq.inria.fr/gamma/ghs3d/ghs.html
} 


\section{Registering the Mesh to a Given Image Modality}

The registration of our biomechanical heart model to a given image modality is necessary in order to fuse multiple information in the same volumetric model. Furthermore, this stage is also a prerequisite before performing the segmentation and tracking in a image modality (MR, 3D US or functional imaging) used clinically. For this reason, this registration stage must be robust but also fairly efficient since it is part of interactive segmentation software.

To achieve the non-rigid registration, we rely on a coarse-to-fine approach proposed by Montagnat et al. [12] that smoothly combines registration and deformable model framework. At a coarse scale, an Iterative Closest Point [3] type of algorithm is applied with successively a rigid, similarity and affine transformations (see section 3.2 ). At a fine scale, the minimization of an internal and external energy allows to obtain more local deformations (see section 3.3). For both methods, it is necessary to determine the closest boundary point to each mesh vertex.

\subsection{Computation of the Closest Boundary Points}

Few authors [7] have previously proposed to segment myocardium images based on a volumetric model. They often rely on interactive segmentation [15] or precomputed distance maps [17] to define the boundary attracting force driving their models. In our approach, the computation of this force at a surface vertex depends not only on the vertex location but also on its normal direction. Different type of forces may be applied depending on the image modality. We have chosen to combine intensity and gradient information with a region-based approach [13] applied to the intensity profile extracted at each vertex in its normal direction. It consists in defining a region with a range of intensity values and then finding its boundary by looking at the voxels of high gradient value. The extent of the intensity profile is decreased in the coarse-to-fine process.

Since there are often occlusions or very noisy parts (for instance, the right ventricle may not be visible or may be greatly biased), we can set the extent of the image interaction (external force) to different values depending on the anatomical regions of our model (determined in section 4): parts which are not seen in the image do not contribute to the external energy.

\subsection{Computation of the Global Transformation}

In our case the initial alignment is simply given by the correspondence of the image and model axes and the rough super-imposition of the ventricles. Then, we iteratively estimate the point matches and the transformation in an Iterative Closest Point-like loop. A rigid transformation is first estimated, then a similarity and finally an affine transformation.

The problem we had with standard least-square similarity and affine estimations in such an iterative loop with real data (like MRI) is that a global minimum is obtained if all model points are matched to the same image point, in which case the transformation is singular (null determinant of the linear part) but the error is null (thus minimized!). In practice, we observed that the least-square estimation was biased toward singular transformations and that the ICP loop often lead to collapse the model into a single voxel. 
To avoid this problem, we use a new affine registration criterion $C$ which is symmetric and forbids singular transformations $\left(x_{i}\right.$ and $y_{i}$ are the matched model and image points, $A$ the affine transformation and $t$ the translation):

$$
C(A, t)=\sum_{i}\left(A \cdot x_{i}+t-y_{i}\right)^{t} \cdot\left(\operatorname{Id}+A^{t} \cdot A\right) \cdot\left(A \cdot x_{i}+t-y_{i}\right)
$$

This criterion can be derived from a statistical formulation and has a closed form solution, detailed in [16]. The scale estimation is different from the leastsquares and the Procrustes ones: it is symmetric (like the Procrustes method [8]) but takes into account the individual matches and not only global properties of the point sets.

The use of this criterion allowed to initialize well the model with a global transformation even in biased images, before fitting the model with a better accuracy using local deformations.

\subsection{Computation of the Local Deformation}

At this stage, our biomechanical model evolves under both the influences of an Internal Energy computed from the physical properties of the organ and an External Energy computed from the image, as defined in the deformable model framework.

Internal Energy. The internal energy is computed with linear elasticity using the Tensor-Mass model [4]. We use the Finite Element Method with linear tetrahedral elements and mass-lumping in a Newtonian differential equation with an explicit time integration scheme. If we want to fit a model built from a heart to an image from another heart, there is no physical basis to the deformation, so we use isotropic elasticity with small Lamé constants to allow greater deformations. But if we want to adapt a model to another image of the same heart, we use anisotropic linear elasticity with Lamé constants, as it physically corresponds to a deformation of the myocardium. And we can also use the anatomical regions to better control the internal energy as we can include different regions (like fat, ischemic zones,... ) modeled with different Lamé constants.

External Energy. At each step of the deformation, we apply forces on the surface nodes of the model along the normal which are proportional to the distance to the match point. The a priori information used is the fact that the points of the mesh we want to match with the image are on the surface of the model and that we know the intensity profile of the boundaries we want to match on these points.

\subsection{Results of the Fitting of the Model to a 3D Image}

We first experimented this method by fitting a canine heart model from dtMRI to the human heart of the VHP (see fig. 2). We used this segmented VHP heart image to define the anatomical regions in the mesh. Although the rotation between the initial mesh and the data was quite important and we were fitting a canine heart mesh to a human image, the rigid to affine to local transformation sequence allowed us to register the model to the image and gave a qualitatively good segmentation of the myocardium. 

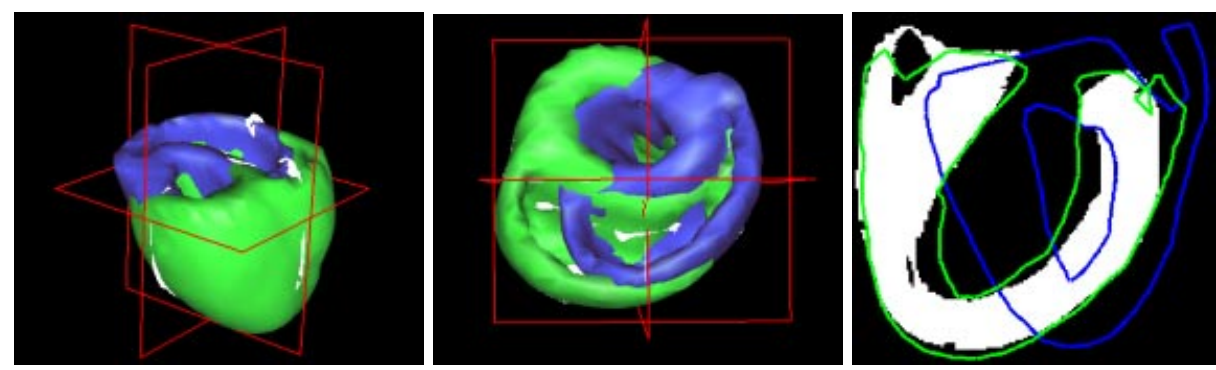

Fig. 2. Fitting of a canine heart model to the Visible Human heart image. dark: before deformation, light: after deformation
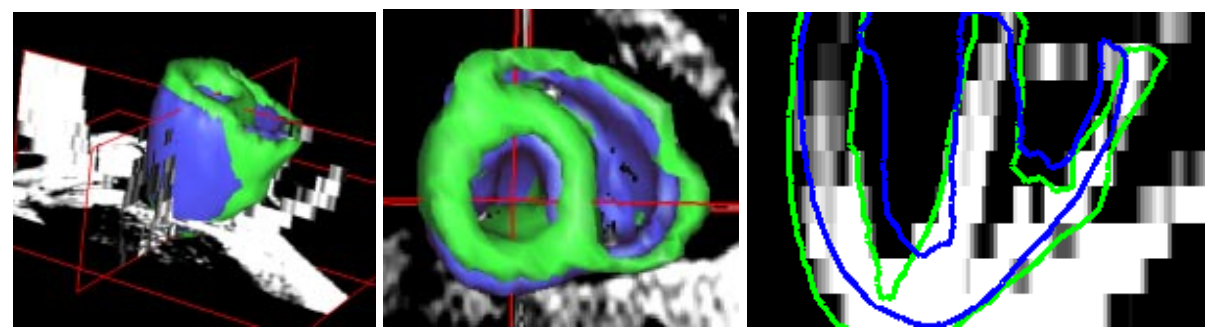

Fig. 3. Fitting of a canine heart model to a human MRI. dark: initial mesh, light: after deformation

Before the transfer of the regions from the image to the mesh by the described rasterization process, we built distance maps from the anatomical image: it allows to assign regions to the parts of the mesh which are not included in the anatomical image due to the limitations of the fitting.

Another experiment was the fitting of the same canine model to a human cardiac MRI. The similarity and the affine transformations could not be computed with the classical least-squares criterion and we had to use the new criterion presented in 3.2 As the right ventricle is very noisy in this image, we only used regions where the myocardium was visible to compute the external forces. MR Images are biased, and a consequence is that the intensity is higher in the apex than around the base. Automatic segmentation of the myocardium in MRI is rather difficult and the presented method gave qualitatively good results (cf. fig. 3).

The fitting of the model in a 3D image takes around $30 \mathrm{~s}$ on a standard PC with a 10000 tetrahedra mesh (due to the anisotropy of the MRI voxels, a finer mesh would not be useful). It is fast enough to be visually controled and interactive.

\section{Assignment of Anatomical Properties}

Our objective is to store several informations originating from different image modalities inside the same volumetric mesh. This information may be quantitative in the case of Diffusion Tensor Magnetic Resonance Imaging (dtMRI) where fiber directions of the myocardium [10]) are extracted. But it can also 



Fig. 4. Left: segmented heart image of the VHP to define anatomical regions. Middle: dtMRI vectorial image to define fiber directions. Right: dorsobasal left epicardial ventricle zone and anisotropy displayed on the final biomechanical model.

be qualitative (semantic) when extracted from precisely segmented anatomical atlases built from the Visible Human Project (VHP) [18] or histology 14. This information may be stored at the vertex or tetrahedron level.

If we assume that the mesh has been precisely registered with a given image modality, we need to find for each tetrahedron (resp. vertex) of the mesh, its corresponding voxels in the volumetric image: this is called the rasterization stage. If the information is to be stored at a vertex, we estimate the attribute with a trilinear or nearest-neighbor interpolation depending if it is a quantitative or qualitative parameter. However, when the information is to be stored at a tetrahedron, we first find the image voxels whose center points are located inside this tetrahedron and then assign either the median or average value depending of the signal to noise ratio.

This set of voxels is found by performing a cascading set of $1 \mathrm{D}$ drawing operations. First, the highest and the lowest horizontal planes intersecting the tetrahedron is determined. Then we find the analytical intersection of its six edges for each intermediate horizontal planes spaced by one voxel height. Thus, we define for each plane, an intersected convex polygon (triangle or quadrilateral) for which we must again find all inside voxels. The same algorithm is applied for filling this polygon by selecting all parallel planes orthogonal to the $\mathrm{X}$ direction for instance. A more complex algorithm would consist in taking into account the partial volume effect by weighting each voxel by its amount of inclusion inside a tetrahedron. However, considering that the size of tetrahedra is larger that the size of a voxel, we think this level of accuracy is not necessary. A result of this process is a biomechanical model of the heart for cardiac image analysis (fig. (4).

\section{Conclusion and Perspectives}

We presented an efficient process to build biomechanical models using a fast volumetric deformation method to fit these models to 3D images. Using a biomechanical model ensures a strong topology constraint and allows to easily include many anatomical and mechanical properties. The deformable model framework is efficient to link biomechanical models and medical images as the image inter- 

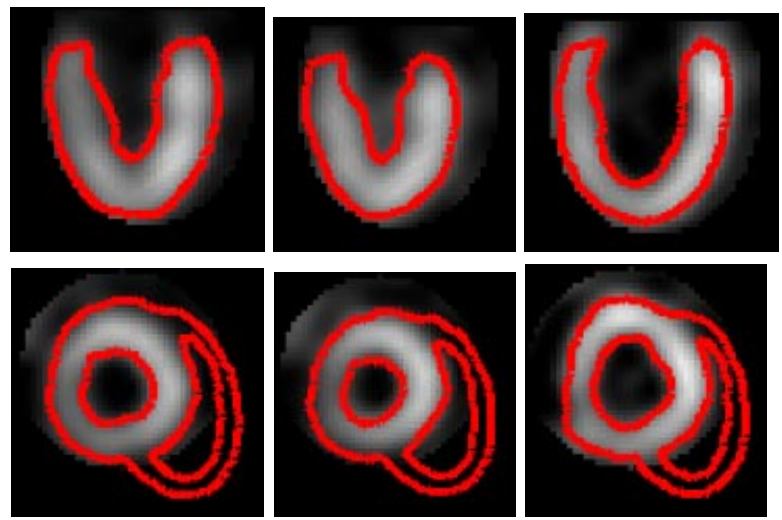

Fig. 5. Tracking of the left ventricle in a 4 D SPECT sequence $\left(8^{*} 64 \times 64 \times 64\right)$. The right ventricle regions have no interaction with the image as they do not appear in it.

acts as a boundary condition in the model evolution. Additional videos can be found on the web2.

A near perspective is to segment sequences of cardiac images by propagating the fitting result obtained a time $t$ as the initialization in the image at time $t+1$. Preliminary results were obtained on a cardiac SPECT sequence of the left ventricle (fig. 5). The evolution of the wall thickness was well represented by this segmentation.

Future work will couple this with the electro-mechanical model presented in [19] to propose a spatiotemporal segmentation process for cardiac images based on a dynamic model of the heart. By using a biomechanical model with an electro-mechanical internal energy, we include a priori knowledge on the geometry and the motion, and we believe it should allow to better recover the cardiac motion from cardiac images.

Acknowledgements: The authors would like to thank Céline Fouard for the distance maps, Prof. Karl Heinz Höhne and his group for the segmented heart data, Dr. Edward Hsu for the Diffusion Tensor images and Philips Research France for the cardiac MRI. This work is a part of the Collaborative Research Action ICEMA 3 .

\section{References}

1. N. Ayache, D. Chapelle, F. Clément, Y. Coudière, H. Delingette, J.A. Désidéri, M. Sermesant, M. Sorine, and J. Urquiza. Towards model-based estimation of the cardiac electro-mechanical activity from ECG signals and ultrasound images. In Functional Imaging and Modelling of the Heart (FIMH'01), number 2230 in Lecture Notes in Computer Science (LNCS), pages 120-127. Springer, 2001.

2. F. Azar, D. Metaxas, and M. Schnall. Methods for modeling and predicting mechanical deformations of the breast under external perturbations. Medical Image Analysis, 6(1):1-27, 2002.

\footnotetext{
${ }^{2}$ http://www-sop.inria.fr/epidaure/personnel/Maxime.Sermesant/gallery.php

${ }^{3}$ http://www-rocq.inria.fr/who/Frederique.Clement/icema.html
} 
3. P.J. Besl and N.D. McKay. A method for registration of 3D shapes. IEEE transactions on Pattern Analysis and Machine Intelligence, 14(2):239-256, 1992.

4. S. Cotin, H. Delingette, and N. Ayache. A hybrid elastic model allowing real-time cutting, deformations and force-feedback for surgery training and simulation. The Visual Computer, 16(8):437-452, 2000.

5. C. Davatzikos. Nonlinear registration of brain images using deformable models. In IEEE Workshop on Math. Methods in Biomedical Image Analysis, pages 94-103, 1996.

6. M. Ferrant, S. Warfield, C. Guttmann, R. Mulkern, F. Jolesz, and R. Kikinis. Registration of $3 \mathrm{D}$ intraoperative $\mathrm{MR}$ images of the brain using a finite element biomechanical model. In MICCAI'00, volume 1935 of Lecture Notes in Computer Science (LNCS), pages 19-28. Springer, 2000.

7. A.F. Frangi, W.J. Niessen, and M.A. Viergever. Three-dimensional modeling for functional analysis of cardiac images: A review. IEEE Trans. on Medical Imaging, 1(20):2-25, 2001.

8. C.R. Goodall. Procrustes methods in the statistical analysis of shape (with discussion). J. Roy. Statist. Soc. Ser. B, 53:285-339, 1991.

9. A. Hagemann, K. Rohr, and H.S. Stiehl. Biomechanically based simulation of brain deformations for intraoperative image correction: coupling of elastic and fluid models. In Medical Imaging 2000 - Image Processing (MI'00), pages 658-667. K. M. Hanson, 2000.

10. E.W. Hsu and C.S. Henriquez. Myocardial fiber orientation mapping using reduced encoding diffusion tensor imaging. Journal of Cardiovascular Magnetic Resonance, 3:325-333, 2001.

11. A. McCulloch, J.B. Bassingthwaighte, P.J. Hunter, D. Noble, T.L. Blundell, and T. Pawson. Computational biology of the heart: From structure to function. Progress in Biophysics \& Molecular Biology, 69(2/3):151-559, 1998.

12. J. Montagnat and H. Delingette. Globally constrained deformable models for 3D object reconstruction. Signal Processing, 71(2):173-186, 1998.

13. J. Montagnat, M. Sermesant, H. Delingette, G. Malandain, and N. Ayache. Anisotropic filtering for model based segmentation of $4 \mathrm{D}$ cylindrical echocardiographic images. Pattern Recognition Letters (in press), 2001.

14. S. Ourselin, E. Bardinet, D. Dormont, G. Malandain, A. Roche, N. Ayache, D. Tande, K. Parain, and J. Yelnik. Fusion of histological sections and mr images: towards the construction of an atlas of the human basal ganglia. In MICCAI'01, volume 2208 of $L N C S$, pages 743-751, Utrecht, The Netherlands, October 2001.

15. X. Papademetris, A. J. Sinusas, D. P. Dione, and J. S. Duncan. Estimation of 3D left ventricle deformation from echocardiography. Medical Image Analysis, $5(1): 17-28,2001$.

16. X. Pennec. Statistical criterions for the rigid, similarity and affine registration of multiple point sets: shape estimation. Research report, INRIA, 2002. To appear.

17. Q.C. Pham, F. Vincent, P. Clarysse, P. Croisille, and I. Magnin. A FEM-based deformable model for the 3D segmentation and tracking of the heart in cardiac MRI. In Image and Signal Processing and Analysis (ISPA'01), 2001.

18. A. Pommert, K.H. Höhne, B. Pflesser, E. Richter, M. Riemer, T. Schiemann, R. Schubert, U. Schumacher, and U. Tiede. Creating a high-resolution spatial/symbolic model of the inner organs based on the visible human. Medical Image Analysis, 5(3):221-228, 2001.

19. M. Sermesant, Y. Coudière, H. Delingette, N. Ayache, and J.A. Désidéri. An electro-mechanical model of the heart for cardiac image analysis, as in [14]. 